\title{
Redes sociales en contextos turísticos costeros: pueblos de apoyo
}

\author{
Aidé Vázquez* \\ Laboratorio de Observación e Investigación Espacial \\ Oscar Frausto Martínez \\ Universidad de Quintana Roo \\ Julia Fraga \\ Centro de Investigación y de \\ Estudios Avanzados del IPN \\ Unidad Mérida
}

\section{Resumen}

Se presenta un trabajo etnográfico realizado con migrantes que habitan en el pueblo de apoyo de Akumal, Quintana Roo. Se emplea el análisis de redes sociales con el fin de identificar y examinar las redes conformadas por dos generaciones de inmigrantes en ese pueblo. La investigación revela las redes de parentesco y de amistad que se producen en los pueblos de apoyo de destinos turísticos de fuerte migración, y permite asociar las redes construidas tanto a nivel individual como de la comunidad. Su aplicación en poblaciones emergentes hace posible comprender el papel de los actores en la gestión de contextos turísticos.

\section{Palabras clave}

Redes sociales, pueblos de apoyo, turismo.

Recibido: 10/06/2014 · Aceptado: 15/12/2014

*Correo electrónico: vazquez.aide@gmail.com• ofrausto@uqroo.edu.mx • fragaje@hotmail.com 


\title{
Social network in tourism contexts: support town
}

\author{
Aidé Vázquez* \\ Laboratorio de Observación e Investigación Espacial \\ Oscar Frausto Martínez \\ Universidad de Quintana Roo \\ Julia Fraga \\ Centro de Investigación y de Estudios Avanzados del IPN \\ Unidad Mérida
}

\begin{abstract}
From an ethnographic work done with migrants in coastal touristic context, the people who live in the support town of Akumal, Quintana Roo. The social network analysis has been used with the purpose of identifying and analyzing the social networks of two generations of migrants in the touristic context of the support town of Akumal, Quintana Roo. The analysis reveals configuration of networks of relationship and kinship that are produced in the support towns of the touristic destinations of strong migration. The need of these analyses allow the association of the networks upon an individual and community level. Its application in emerging communities allows an approaching in the understanding of the roles of the actors in the management of touristic context.
\end{abstract}

\section{KEY WORDS}

Social network, support town, tourism.

*E-mail: vazquez.aide@gmail.com• ofrausto@uqroo.edu.mx• fragaje@hotmail.com 


\section{Introducción}

La naturaleza de la actividad turística es de tipo económico, social y cultural; implica transformaciones en las condiciones de vida de sectores de trabajadores, así como la modificación, creación o desaparición de estructuras ambientales, sociales y culturales (Hogan, 2002; Monterrubio, 2011).

Este fenómeno comenzó en México en 1968, cuando el gobierno decidió seguir los pasos de España, que había encontrado en el turismo la respuesta a sus problemas económicos. Se promovió un programa integral de centros turísticos, cuya primera fase incluyó estudios para identificar los territorios donde se establecerían los centros integralmente planeados (CIP). Cancún fue seleccionado como el primer centro turístico y la primera ciudad integralmente planeada, a él le seguirían Los Cabos, Puerto Escondido, Huatulco e Ixtapa (Castro, 2007; Fraga, 2012; Talledos, 2012). Entre sus propósitos se encontraba impulsar la economía regional, crear empleos y dirigir la migración interna hacia los destinos turísticos (Wilson, 2008), lo cual supuso el inicio de los trabajos de infraestructura turística y transporte, así como la conformación de los primeros campamentos de trabajadores y, en consecuencia, la urbanización de las zonas turística y poblacional, esta última concebida como espacio para proveer de servicios y vivienda a los trabajadores.

Los migrantes han sido elemento fundamental de la mano de obra en la industria del turismo, pues el entorno laboral de esta ofrece a los recién llegados la posibilidad de integrarse a la sociedad de manera personal, profesional y social (Janta et al., 2011). Sin embargo, la presencia de inmigrantes en zonas costeras sensibles a los procesos de urbanización (crecimiento) y turismo (prácticas) es determinante en los efectos de las acciones antropogénicas en esas costas vulnerables (González y Palafox, 2006; Sherbinin et al., 2007), pues al erigir ciudades turísticas costeras se modifica el territorio y se evidencia que este proceso responde a la demanda, ante la ausencia de un desarrollo estructurado que considere en primer término a la sociedad (González y Palafox, 2006).

Es cada vez más necesario estudiar y caracterizar la industria turística en Quintana Roo, en sus afectaciones sociales y de entorno, a fin de tener una mayor comprensión sobre el desarrollo y los mecanismos internos de funcionamiento de los destinos turísticos. 
Por ello se llevó a cabo el presente trabajo etnográfico con migrantes que viven en el pueblo de apoyo de Akumal, Quintana Roo, en un contexto turístico costero. Se identificaron y estudiaron las redes sociales conformadas por dos generaciones de inmigrantes.

El modelo de análisis de redes sociales descubre las redes de parentesco y de amistad que se producen en los destinos turísticos de fuerte migración y permite la asociación de las redes según los niveles individual y de comunidad, lo cual, a su vez, facilita la comprensión del papel de los actores en la gestión de comunidades emergentes, teniendo como punto de partida las repercusiones del turismo.

\section{Revisión de literatura}

El carácter multidimensional del turismo ha motivado a los académicos a realizar estudios desde las perspectivas económica, sociocultural y ambiental. La primera se centra en la oferta y la demanda turísticas, el origen y el destino de los visitantes, así como en la industria y en la infraestructura del sector, todo esto como sinónimo de las "bondades" del turismo. Por su parte, los aspectos sociales y ambientales han sido abordados a partir de sus impactos y como consecuencia del desequilibrio inducido por el turismo en cuanto actividad económica predominante (Monterrubio, 2011).

Los estudios sobre migración en contextos turísticos han enfatizado el papel de los migrantes como trabajadores y su contribución en la economía de los destinos, problemática que tratan desde un enfoque psicosocial, dirigiendo su atención a las motivaciones, el comportamiento y las experiencias de los migrantes (Joppe, 2012; Janta et al., 2011) y a la percepción de los migrantes sobre los destinos turísticos (Carte et al., 2010).

La creación de las nuevas ciudades costeras va acompañada de procesos de migración, urbanización y turismo que siguen un modelo de desarrollo turístico ajeno a la perspectiva social y cultural del lugar (González y Palafox, 2006).

Se ha identificado que la industria turística de Quintana Roo se concentra en las localidades del corredor de la Riviera Maya, principalmente Cancún, Playa del Carmen y Tulum, y ahí se ha formado un contexto regional único para la 166 migración (Carte et al., 2010). Lo anterior ha propiciado una división espacial 

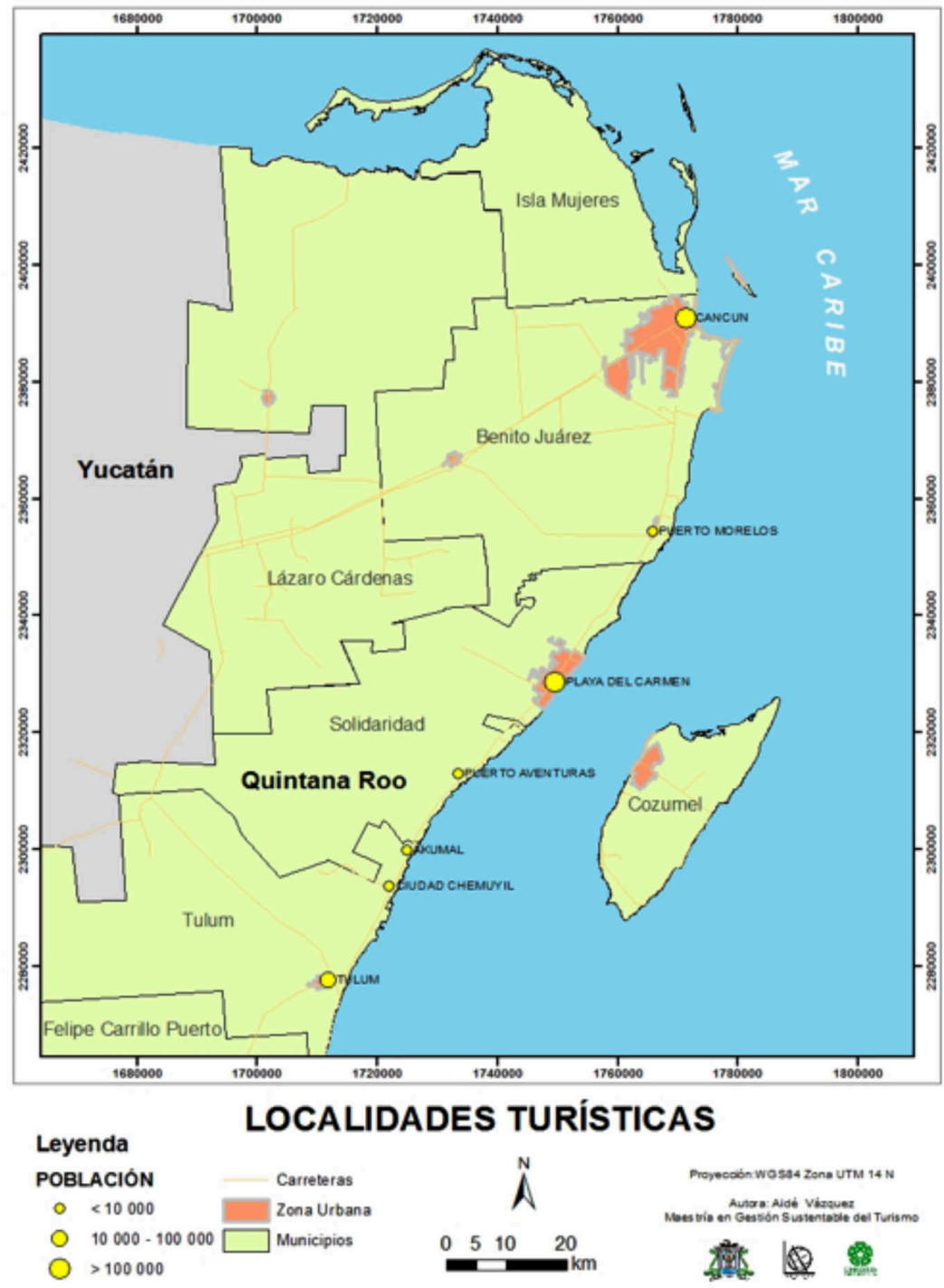

Figura 1. Localidades turísticas y pueblos de apoyo 
entre las áreas turísticas y las de residencia de los trabajadores (Manuel-Navarrete, 2012); estas últimas han sido etiquetadas como comunidades de servicios (Pi-Sunyer y Brooke, 2005), ciudades gemelas (Córdoba y García, 2003), pueblos de servicios (Manuel-Navarrete, 2012) y pueblos de apoyo (Fraga, 2012).

La vocación turística de Quintana Roo queda de manifiesto al constatar que registra la mayor tasa de crecimiento turístico y demográfico del país, ya que ha impulsado prácticas fundamentadas en un turismo sustentable y el desarrollo económico de la región; sin embargo, ha predominado una actividad turística masiva de sol y playa y de cruceros. Como resultado, a lo largo de toda la costa del Caribe mexicano se han erigido complejos turísticos que requieren fuerza laboral para respaldar su desarrollo turístico, lo que ha derivado en la conformación de pueblos de apoyo habitados por inmigrantes.

El estudio se llevó a cabo en el litoral oriental de la costa de la Península de Yucatán, que va de Cancún a Tulum, donde se han formado pueblos de apoyo como Puerto Morelos, Playa del Carmen, Puerto Aventuras, Akumal y Chemuyil, integrados en el corredor turístico Riviera Maya (figura 1). Estos pueblos se han asentado alrededor de los complejos turísticos, del otro lado de la carretera federal, y son habitados en su mayoría por inmigrantes trabajadores provenientes de comunidades rurales que constituyen la fuerza de trabajo que sostiene la actividad turística (Dachary y Arnaiz, 2009; Re Cruz, 2006; Fraga, 2012).

\section{Metodología}

En la gestión del turismo de un lugar son determinantes los actores que intervienen en él y las relaciones que establecen entre sí. Su identificación y caracterización son complejas, y el tratamiento de sus interacciones demanda una mayor atención, razón por la cual aquí se parte del análisis de redes sociales en contextos turísticos.

El trabajo de campo se llevó a cabo en Akumal (en maya, lugar de tortugas), considerado el primer enclave turístico de la costa, pero que antes de explotar su vocación turística (1940) se dedicaba a la comercialización de la copra. Sin embargo, en 1950 esta producción se había debilitado y en 1955 el sitio fue devastado por el huracán Janet. Con estos antecedentes, en 1959 se inicia la privatización 168 de su territorio, cuando el Club de Exploración y Deportes Acuáticos de México 
(CEDAm) comienza los trabajos de rescate del barco El Matancero (que en el siglo xviII había naufragado cerca de ahí) y cuando un particular adquiere la concesión de tierras de la costa y emprende el desarrollo de un complejo turístico que se afianzó en 1975, con la consecuente atracción de inmigrantes. Akumal es una de las localidades con mayor población (1 310 habitantes en 2010) del municipio de Tulum (INEGI, 2010); se encuentra en el corredor Riviera Maya, a 37 km de Playa del Carmen y a $26 \mathrm{~km}$ de Tulum, sobre la carretera federal 307. Actualmente tiene 24 hoteles con 2568 habitaciones (Vázquez, 2007). Se extiende en $155056.89 \mathrm{~m}^{2}$ de litoral costero, abarcando desde la Caleta Yalku hasta el hotel Bahía Príncipe y al otro lado de la carretera el pueblo de apoyo Akumal.

El periodo de levantamiento comprendió de enero a abril de 2013, y se utilizó la técnica de bola de nieve; de acuerdo con Maya (2001), al tratarse de un grupo disperso, la técnica de selección en cadena o bola de nieve es la indicada para poblaciones inmigrantes.

Mediante esta técnica y con una encuesta en el hogar, se identificó la primera generación de conformidad con los siguientes criterios: a) que el movimiento migratorio se hubiera producido entre 1974 y 1980, y b) que se mantuviera la constancia de habitar en el pueblo de apoyo. La segunda generación corresponde a los hijos e hijas que han crecido en la comunidad. Según Rumbaut (2006), la generación de migrantes puede ser estudiada desde dos perspectivas: por cohorte demográfica y por parentesco -es decir, por su pertenencia a la misma genealogía-. Se identificaron y encuestaron 14 residentes de primera generación y 11 de segunda. Así, el análisis etnográfico se realizó con base en fuentes primarias de observación, apoyadas en las encuestas a actores clave.

En la explicación de estos procesos migratorios de cambio, articulación y reconstrucción de la sociedad de estudio se recurrió, como ya se dijo, al análisis de redes sociales -que trata los vínculos en situaciones de desposesión de lazos de pertenencia y en momentos de vulnerabilidad social-, el cual nos permite reconocer a sus protagonistas y sus historias (Gómez, Molina y Roca, 2008). Para Lozares (1996: 108), las redes sociales son "un conjunto bien delimitado de actores -individuos, grupos, organizaciones, comunidades, sociedades globales, etc.- vinculados unos con otros a través de una relación o un conjunto de relaciones sociales". Con el tipo de análisis utilizado es posible identificar las estructuras sociales conformadas a partir de la migración y su proceso de 
adaptación, lo cual es de gran utilidad para comprender las relaciones entre los actores y entender sus interacciones individuales y a nivel comunidad en el pueblo de apoyo de Akumal.

Diversas disciplinas incorporan en sus etapas metodológicas el análisis de redes sociales, principalmente en las investigaciones interesadas en conocer la estructura de redes sociales de una comunidad o grupo en concreto, la relación de personas entre sí, o la vinculación de colectivos o instituciones. Gualda (2005: 21) reconoce que "uno de los elementos que integran nuestra realidad es precisamente la interacción, vínculo o relación que se produce entre actores individuales y colectivos", es decir, la razón de ser del análisis de redes sociales, que encuentra su complemento en el examen del contexto en el que se produce la relación social.

Algunos estudios sobre análisis de redes sociales han ofrecido un primer acercamiento a los modelos de análisis del sistema turístico local (los actores y sus relaciones), ubicando a los actores turísticos locales y sus relaciones como piezas clave en el nuevo modelo turístico. Desde este punto de vista, los actores locales, en un territorio determinado, son los protagonistas que impulsan el crecimiento económico a través del turismo, evidenciado la correlación entre el nivel de desarrollo turístico de un territorio y las características de las relaciones entre los actores involucrados en él (Merinero, 2010: 278).

Merinero y Pulido establecen que "El conocimiento sobre los actores turísticos del territorio y sus relaciones es un elemento básico para la gestión activa de cualquier destino turístico, pues éstos tienen una importancia especial en las estrategias y las acciones que deben emprenderse para adaptarse a las nuevas condiciones del contexto.” (2009: 173).

\section{Resultados}

Se presentan las características de los actores en el entorno turístico del pueblo de apoyo de Akumal y las redes familiares y de amistad (por generación) que han permeado en la comunidad. 


\section{Primera generación}

Se identificó y encuestó a la primera generación de inmigrantes (11 hombres y 3 mujeres de entre 49 y 85 años de edad al momento de la encuesta), quienes han sido parte de la comunidad por más de 30 años, al haber inmigrado entre 1974 y 1983; son originarios de Yucatán, sobre todo de Kantunil (43\%), Ticul (29\%), Valladolid (14\%), Tizimín (7\%) y Sotuta (7\%), por lo que se trata de una migración (rural-destino turístico) interestatal.

Su estado civil es predominantemente casados/as (71 \%), con un promedio de 5.8 hijos/as por hogar; en su mayoría profesan la religión católica (93\%). Antes de migrar, $86 \%$ de ellos había cursado solo el nivel educativo básico en una institución pública, situación que no se ha modificado. Todos afirmaron entender "la [lengua] maya”, 93 \% hablarla, pero ninguno escribirla; además, $36 \%$ entiende y habla inglés, pero ninguno lo escribe.

En cuanto al aspecto laboral anterior a la migración, 71 \% se encontraba trabajando como campesino, panadero, zapatero o comerciante. No obstante, al momento de migrar su actividad pasó a la industria turística, como ayudantes de cocina, camaristas, garroteros, encargados de mantenimiento, meseros o marineros; en la actualidad, a medida que la edad aumenta, la proporción de empleados disminuye y se engrosan las categorías de trabajador por cuenta propia, pensionados y amas de casa ( $60 \%$ ), lo cual contrasta con el caso de sus hijos e hijas, que en su mayoría están desempeñándose en el sector turístico (75 \%).

El modelo de análisis aquí utilizado nos permite visualizar la distribución de la red de relaciones familiares a nivel individual, así como la red de apoyo que han conformado y su rol en la estructura social, la cual es principalmente interna (hacia la comunidad). También se observa la integración de un capital social que les brinda información (de oportunidades laborales y sociales), recursos y respaldo emocional.

Así, las redes sociales son un factor fundamental al tomar la decisión de emigrar, así como al llegar al nuevo entorno laboral y social. Las redes sociales de la primera generación son primordialmente de parentesco (64 \% manifestó contar con familia). La distribución de las redes familiares en el pueblo de apoyo se observa en la figura 2. Cabe destacar que $93 \%$ tiene redes familiares en la Riviera Maya. 
La figura muestra las redes sociales en el pueblo de apoyo de Akumal; sobre la mancha urbana de la comunidad se encuentra remarcada, con un tono oscuro, la ubicación de la primera generación; las flechas indican su distribución en el interior y en el exterior -en Quintana Roo: Tulum, Chemuyil, Cozumel y Playa del Carmen, y en Yucatán: Ticul, Kantunil y Sotuta-. Se trata de una red de parentesco interna a la comunidad. La enumeración corresponde al orden de encuesta de los inmigrantes.

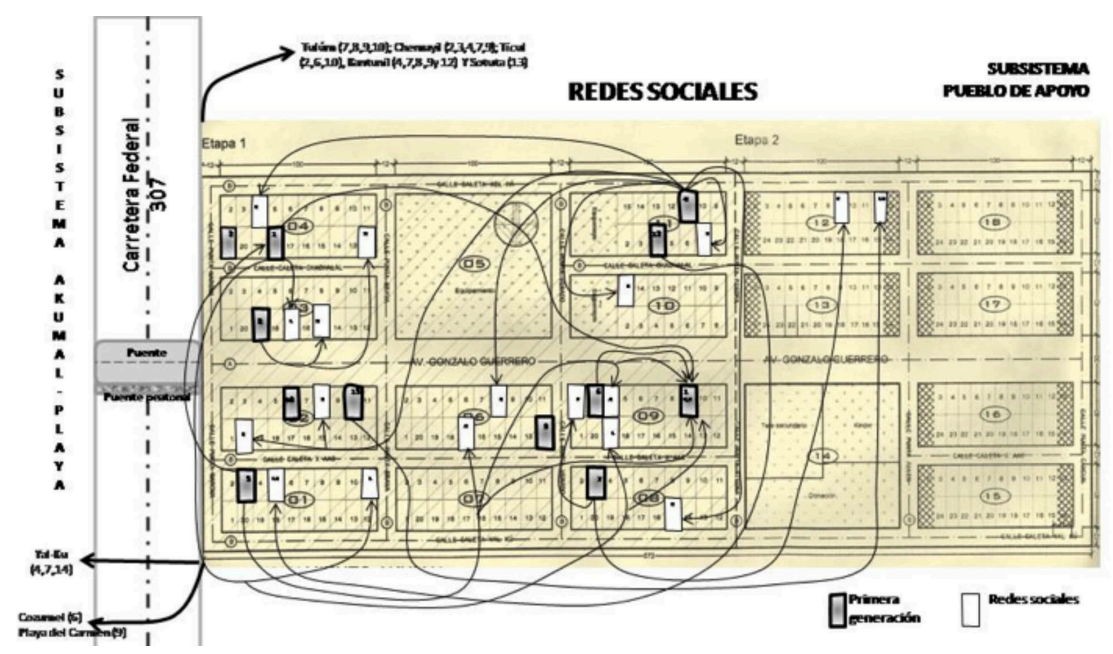

Fuente: Encuesta de migración y turismo en Akumal (2013).

Figura 2. Primera generación de inmigrantes: distribución de las redes sociales

Segunda generación

La segunda generación nació en el estado de Yucatán, de manera señalada en Kantunil (36\%), Valladolid (27\%), Ticul (18\%) y Sotuta (9\%). Sin embargo, al interrogar sobre el lugar respecto del cual se sienten originarios, la respuesta es el estado de Quintana Roo, y más específicamente de Akumal (64\%); el hecho de sentirse de Kantunil y Ticul se vincula con la edad en la que se integraron definitivamente a Akumal, de 15 años de edad en adelante, mientras que quienes se 
asumen como oriundos de Quintana Roo llegaron siendo niños (desde los quince días de nacidos hasta una edad máxima de cinco años).

Con respecto al estado civil, $46 \%$ está casado/a; $18 \%$ son madres solteras. El promedio de hijas/os por hogar es de 2.3 y 73 \% pertenece a la religión católica. Han cursado el nivel preparatorio o bachillerato $36 \%$, la secundaria $36 \%$ y la primaria $27 \%$, en una institución pública. Sobre el dominio de la lengua indígena, todos reconocen "la maya" como propia, no obstante, 73 \% la entiende, $36 \%$ la habla y ninguno la escribe; finalmente, destaca que $73 \%$ entiende y habla inglés, y un porcentaje menor (35\%) lo escribe.

En este caso, el modelo analítico reveló la configuración y distribución de la red de relaciones familiares y de amistades en el plano individual, así como la red de apoyo que han conformado y su papel en la estructura social, que en la segunda generación es en esencia externa a la comunidad.

Las redes sociales de la segunda generación se distribuyen ante todo en la familia residente en el pueblo de apoyo, como puede observarse en la figura 3, además de la red de amistades que se ha establecido en Akumal, Chemuyil, Tulum, Puerto Aventuras, Playa del Carmen y Cozumel.

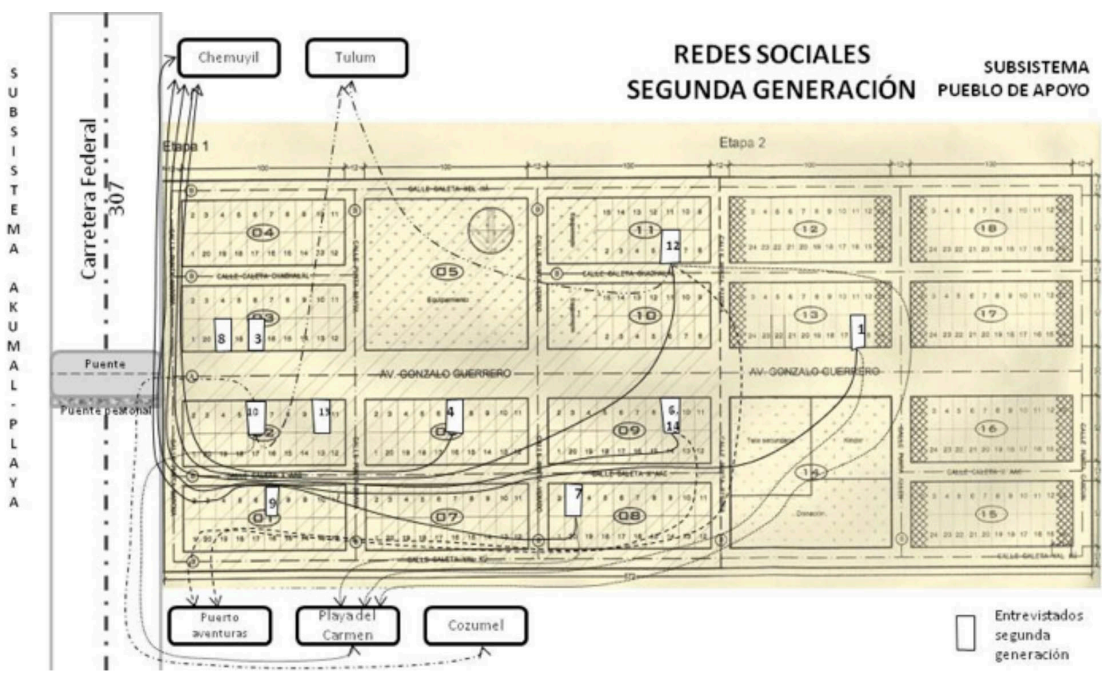

Fuente: Encuesta de migración y turismo, Akumal (2013)

FiguRa 3. Segunda generación: distribución de las redes sociales 
La figura 3 facilita de comprensión del análisis de redes sociales de la segunda generación asentada en la marcha urbana del pueblo de apoyo de Akumal; con cuadros en tono claro se ubican los hogares de los encuestados y con las flechas su distribución en el interior de la comunidad (correspondiente a las redes familiares) y en el exterior (redes de amigos).

\section{Conclusiones}

El análisis de redes sociales aporta elementos para explicar la realidad social, al estudiar la interacción, el vínculo o las relaciones que se producen entre los actores individuales y colectivos; este análisis considera la situación contextual (contextos sociales, culturales, geográficos y económicos, entre otros) en la que se produce la relación social, y su valor analítico permite la comprensión de algunos fenómenos sociales (Gualda, 2005). La reflexión de Gualda fundamenta el nivel organizacional y grupal en economías locales, así como en procesos de adaptación y calidad de vida a partir del estudio de la familia y del sentido de comunidad; también trata la forma en que los grupos se estructuran y su forma de generar el capital social (en el que intervienen las emociones y la cercanía de amigos y familia es indispensable).

En nuestra investigación puede concluirse que la migración interestatal en Quintana Roo se inició a principios de los setenta con los cambios en el uso del territorio y en la economía del país. Después de 39 años, y tras el examen y la comprensión de las implicaciones de lo anterior en la estructura social del pueblo de apoyo de Akumal, por medio del análisis descriptivo es posible exponer una visión desde la perspectiva de sus inmigrantes.

Fue posible caracterizar a los migrantes del pueblo de apoyo de Akumal e identificar su función y percepción de sus redes sociales, además de representar (a través de un modelo) la configuración de sus redes.

El principal factor de consolidación de los centros urbanos-pueblos de apoyo es el desarrollo de la infraestructura turística, la cual incentiva la inmigración. Para la primera generación, las redes sociales fueron determinantes en la decisión de emigrar, pues, como establece Fraga (2012), la migración está basada fundamentalmente en las redes de amistad y en los lazos de parentesco. Para los varones, el motivo para inmigrar es el factor económico, y, para las mujeres, 
la familia. La elección del lugar responde a las redes sociales y las oportunidades laborales. Se trata, entonces, de una migración escalonada. Las redes sociales de la primera generación son de parentesco -establecidas predominantemente en Akumal-, y este fenómeno se ha extendido a la Riviera Maya, dada la movilidad de los miembros de la familia ya sea por trabajo, por matrimonio o por estudios.

En términos culturales, a pesar de los cambios propiciados por el turismo, la fiesta del pueblo de origen se mantiene sólida y el papel de las redes sociales mediante parentesco y amistad se conservan a través de los gremios en los lugares de origen.

En la segunda generación, las redes sociales son de parentesco y de amistad, establecidas primordialmente en Akumal, pero su construcción se ha extendido a la Riviera Maya (en particular a Chemuyil, Tulum, Puerto Aventuras y Playa del Carmen).

\section{Fuentes citadas}

Carte, L. et al. (2010). "Experiencing Agricultural Failure: Internal Migration, Tourism and Local Perceptions of Regional Change in the Yucatan”. Geoforum, 41 (5), 700-710.

Castro, U. (2007). "El turismo como política central de desarrollo y sus repercusiones en el ámbito local: algunas consideración referentes al desarrollo de enclaves turísticos en México”. TURyDES. Revista de Investigación en Turismo y Desarrollo Local, 1 (1).

Córdoba, O. y A. García (2003). “Turismo, globalización y medio ambiente en el Caribe mexicano”. Investigaciones Geográficas, 52, 117-136.

Dachary, A. y S. Arnaiz (2009). "Pueblos originarios y turismo en América Latina”. Estudios y Perspectivas en Turismo, 18 (1), 69-91.

Fraga, J. (2012). "Migración y turismo en la Riviera Maya a través de dos pueblos del Mundo Maya”, en G. Marín, A. García y M. Daltabuit (coords.). Turismo, globalización y sociedades locales en la península de Yucatán, México. Tenerife: Pasos, Revista de Turismo y Patrimonio Cultural (Pasos Edita, 7), 45-74. 
Gómez, S., J. Molina y B. Roca (2008). "Redes sociales y antropología. Introducción”, en A. Leizaola y J. Hernández (coords). Miradas, encuentros y críticas antropológicas. Donostia: Ankulegi Antropologia Elkartea (XI Congreso de Antropología de la FAAEE), 21-26.

González, M. y A. Palafox (2006). "Producción del espacio turístico en ambientes sensibles. Isla de Cozumel, México, y Cayo Las Brujas, Cuba”. Estudios y Perspectivas en Turismo, 15 (2), 149-178.

Gualda, E. (2005). "Pluralidad teórica, metodológica y técnica en el abordaje de las redes sociales: hacia la 'hibridación’ disciplinaria”. Redes. Revista Hispana para el Análisis de Redes Sociales, 9, 1-24.

Hogan, D. (2002). "Movilidad poblacional, sustentabilidad ambiental y vulnerabilidad social: una perspectiva latinoamericana”, en E. Leff et al. (comps.). La transición hacia el desarrollo sustentable. Perspectivas de América Latina y el Caribe. México: Instituto Nacional de Ecología/ Universidad Autónoma Metropolitana/Programa de Naciones Unidas para el Medio Ambiente, 161-185.

Inegi (2010). Censo de Población y Vivienda 2010. Principales resultados por localidad [en línea]. Instituto Nacional de Estadística y Geografía. Disponible en: http://www.inegi.org.mx/sistemas/consulta_resultados/ iter2010.aspx?c $=27329 \& \mathrm{~s}=$ est $[2012,3$ de noviembre $]$.

Janta, H. et al. (2011). "Migrant Relationships and Tourism Employment”. Annals of Tourism Research, 38 (4), 1322-1343.

Joppe, M. (2012). "Migrant Workers: Challenges and Opportunities in Addressing Tourism Labour Shortages”. Tourism Management, 33 (3), 662671.

Lozares, C. (1996). “La teoría de redes sociales”. Papers, 48, 103-126.

Manuel-Navarrete, D. (2012). "Entanglements of Power and Spatial Inequalities in Tourism in the Mexican Caribbean” [en línea]. desiguALdades.net (Working Paper, 17). Disponible en: http://www.desigualdades.net/ Resources/Working_Paper/17_WP_Manuel-Navarrete_online.pdf

Maya, I. (2001). "Sesgos de medida y problemas de muestreo en las encuestas de poblaciones inmigrantes”. Metodología de Encuestas, 3 (2), 197-213. 
Merinero, R. (2010). "Desarrollo local y análisis de redes sociales: el valor de las relaciones como factor del desarrollo socioeconómico". Redes. Revista Hispana para el Análisis de Redes Sociales, 18 (11), 277-304.

Merinero, R. y J.I. Pulido (2009). "Desarrollo turístico y dinámica relacional. Metodología de análisis para la gestión activa de destinos turísticos”. Cuadernos de Turismo, 23, 173-193.

Monterrubio, J. (2011). Turismo y cambio sociocultural. Una perspectiva conceptual. México: Plaza y Valdés.

Pi-Sunyer, O y R. Brooke (2005). "Tourism, Environmentalism, and Cultural Survival in Quintana Roo”, en L. King y D. McCarthy (eds.). Environmental Sociology: from Analysis to Action. Lanham/Londres: Rowman \& Littlefield, 43-60.

Re Cruz, A. (2006). "Turismo y migración entre los mayas de Yucatán. Las nuevas milpas de Chan Kom”. Revista Española de Antropología Americana, 36 (1), 149-162.

Rumbaut, R. (2006). "Edades, etapas de la vida y cohortes generacionales: un análisis de las dos primeras generaciones de inmigrantes en Estados Unidos”, en A. Portes y J. DeWind (coords.). Repensando las migraciones. Nuevas perspectivas teóricas y empíricas. México: Miguel Ángel Porrúa/Universidad Autónoma de Zacatecas/Instituto Nacional de Migración, 361-409.

Sherbinin, A. et al. (2007). "Population and Environment". Annual Review of Environment and Resources, 32, 345-373.

Talledos, E. (2012). "La imposición de un espacio: De La Crucecita a Bahías de Huatulco”. Revista Mexicana de Ciencias Políticas y Sociales, LVII (216), 119-142.

Vázquez, A. (2007). "Indicadores de la Agenda 21 para el turismo Mexicano: caso de estudio Akumal, Quintana Roo”. Tesis de licenciatura en Turismo. Universidad de Quintana Roo.

Wilson, T. D. (2008). "Economic and Social Impacts of Tourism in México". Latin American Perspectives, 35 (3), 37-52. 